\title{
Technè
}

La science au service de l'histoire de l'art et de la préservation des biens culturels

46 | 2018

Science et conservation

\section{Des collections privées aux musées. La restauration des peintures à Paris au XIX siècle}

From private collections to museums. The restoration of paintings in 19thcentury Paris

\section{Barbara Jouves}

\section{(2) OpenEdition}

1 Journals

\section{Édition électronique}

URL : http://journals.openedition.org/techne/284

DOI : $10.4000 /$ techne.284

ISSN : 2534-5168

\section{Éditeur}

C2RMF

\section{Édition imprimée}

Date de publication : 1 décembre 2018

Pagination : 6-10

ISBN : 978-2-11-152829-1

ISSN : $1254-7867$

Référence électronique

Barbara Jouves, «Des collections privées aux musées. La restauration des peintures à Paris au xix ${ }^{\mathrm{e}}$ siècle », Technè [En ligne], 46 | 2018, mis en ligne le 19 décembre 2019, consulté le 22 juillet 2020. URL : http://journals.openedition.org/techne/284; DOI : https://doi.org/10.4000/techne.284

\section{(c) (i) $\odot$}

La revue Technè. La science au service de l'histoire de l'art et de la préservation des biens culturels est mise à disposition selon les termes de la Licence Creative Commons Attribution - Pas d'Utilisation Commerciale - Pas de Modification 4.0 International. 


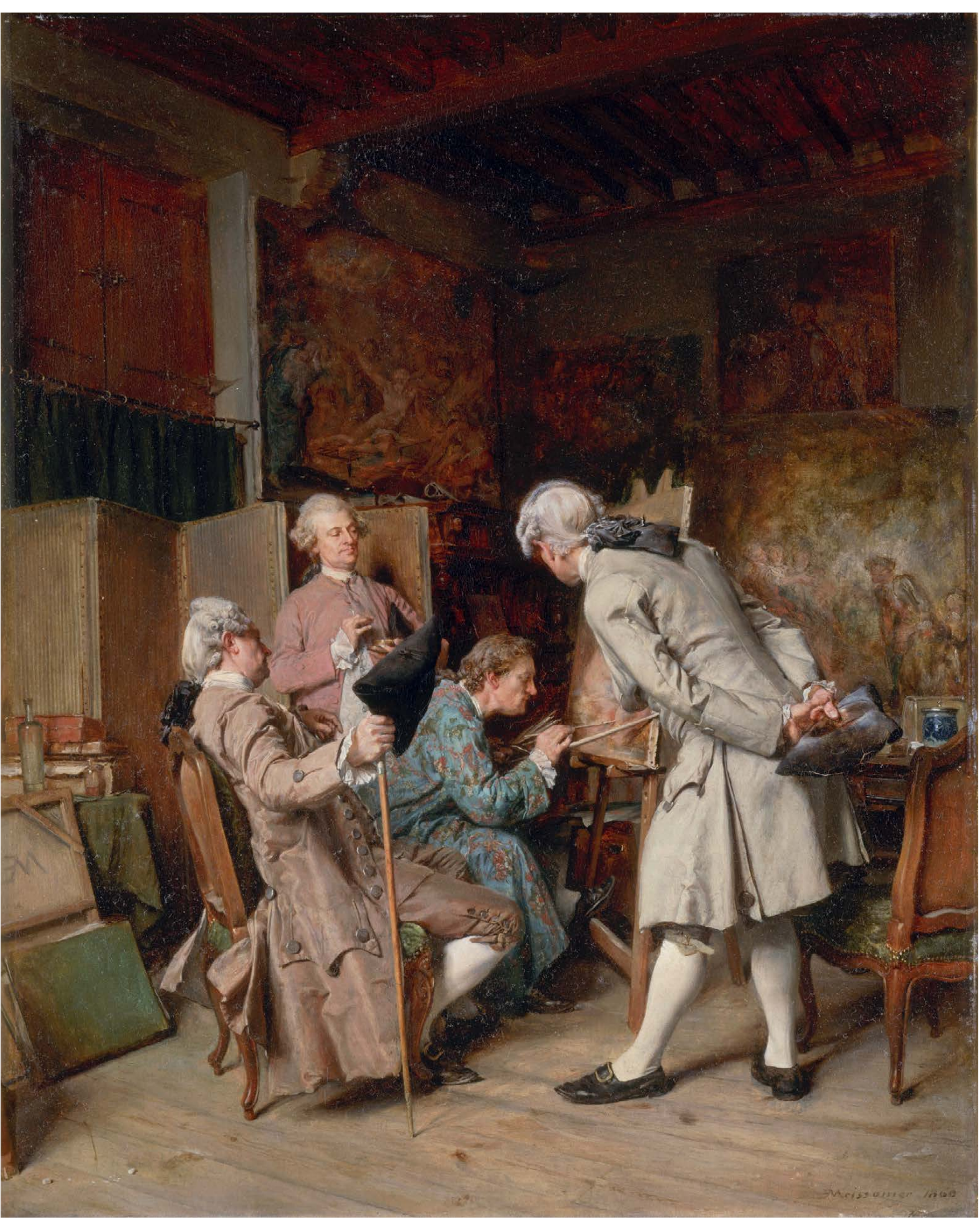

Fig. 1. Ernest Meissonnier, Les amateurs de peinture, dit aussi Un peintre, 1860 , huile sur bois $(35,5 \times 28,5 \mathrm{~cm})$,

Paris, musée d'Orsay. @ RMN-Grand Palais (musée d’Orsay)/Hervé Lewandowski. 


\section{Des collections privées aux musées. La restauration des peintures à Paris $\mathrm{au} \mathrm{XIX}^{\mathrm{e}}$ siècle}

From private collections to museums.

The restoration of paintings in 19th-century Paris

Résumé. Préoccupés par la conservation de leurs collections de peintures, les amateurs d'art parisiens font appel, entre 1789 et 1870, aux restaurateurs de tableaux, ces derniers relevant d'une profession qui, à l'époque, se définit indépendamment de celles du marchand, de l'expert ou même du peintre. Si le restaurateur intervient sur les cuvres du particulier, il joue, par ailleurs, pour l'amateur, un rôle de guide dans sa connaissance, voire dans son apprentissage, des procédés picturaux. Progressivement, ce rapport à la matérialité de l'œuvre contribue à intégrer le collectionneur au sein des commissions muséales en tant que conseiller, avant d'acquérir un statut privilégié au sein du musée à partir des années 1860 par le legs de ses æuvres.

Mots-clés. Amateur, collection, connaisseur, conservation, expert, marchand, marché de l'art, matérialité, musée, patrimoine, peinture, restauration, transmission.
Abstract. Concerned about the conservation of their art collections, in the years between 1789 and 1870, Parisian art lovers called upon the services of painting restorers, who, at the time, belonged to a profession considered quite separate from that of art dealer, expert or even painter. While the restorer worked on paintings belonging to private collectors, he also acted as a guide for the latter, broadening their knowledge of - or even teaching them about - pictorial techniques. This understanding of the materiality of artworks gradually contributed to collectors being invited into museum committees as advisors, before they acquired a privileged status in museums, from the 1860s onwards, by bequeathing their collections.

Keywords. Amateur, collection, connoisseur, conservation, expert, dealer, art market, materiality, museum, heritage, painting, restoration, transmission.
Si l'histoire des restaurations en France a fait l'objet d'études approfondies ces dernières années ${ }^{1}$ et, encore récemment, de journées de réflexion ${ }^{2}$, la recherche concernant les restaurations des peintures de collections privées au XIX ${ }^{\mathrm{e}}$ siècle reste à défricher. Leur nombre à Paris ne cesse pourtant de croître à cette période et, selon les recensements des guides de l'époque, qui n'indiquent encore qu'une partie de ces collections, vingt-six cabinets de tableaux sont conseillés aux voyageurs en $1790^{3}$, contre cent soixante-dix-sept en $1861^{4}$. Au vu des archives privées ou de celles des musées nationaux, des Almanachs du commerce, des catalogues de ventes aux enchères parus à Paris, de la littérature technologique, de la presse artistique et de l'analyse des œuvres restaurées au musée du Louvre jusqu'en 1870, il apparaît que la figure du restaurateur prend durant cette période une nouvelle dimension. Dans cette histoire sociale de l'art, déterminée par rapport aux œuvres et à la volonté du collectionneur de les conserver, la restauration est une profession qui se redéfinit. Si le restaurateur joue un rôle notable dans la volonté de transmettre un savoir sur l'histoire et la lecture matérielle des peintures, à l'instar du marchand de tableaux au XvIII ${ }^{\mathrm{e}}$ siècle $^{5}$, devient-il pour autant un intermédiaire privilégié entre l'œuvre et le collectionneur au XIX ${ }^{\mathrm{e}}$ siècle ? Et quel est son rôle auprès de l'amateur, ce dernier occupant progressivement une place reconnue, d'une part au sein des commissions muséales, d'autre part, en tant que donateur ? Sur ces questions, l'article souhaite apporter quelques données nouvelles, nourries d'une recherche plus approfondie en cours ${ }^{6}$.

\section{L'œuvre et la collection : une nouvelle approche de la matière}

Bien loin de la «maudite race que celle des amateurs ${ }^{7}$ » qualifiée ainsi par Denis Diderot en 1767 , le xıx ${ }^{\mathrm{e}}$ siècle voit se dessiner une nouvelle approche du collectionneur, non pas tant dans le mode d'acquisition de l'objet, dont le marché de l'art a installé les préceptes désormais établis ${ }^{8}$, que dans la manière de concevoir et d'entretenir l'objet. De l'écrin privé à l'espace de représentation, la collection continue à s'imposer comme 
l'un des symboles d'une sociabilité exemplaire, s'inscrivant dans une volonté grandissante d'acquérir des œuvres conformes au goût de l'époque ${ }^{10}$.

À Paris, le nombre d'amateurs de tableaux (fig. 1) connaît une forte croissance, ce dont témoignent les ventes aux enchères publiques de collections parisiennes : en 1789, il est recensé vingt et une ventes comprenant des peintures, en 1820 cinquante, en 1850 cent six et en 1870 cent cinquanteneuf ${ }^{11}$. Les livrets de ces ventes étaient devenus, dès la seconde moitié du XvirI ${ }^{\mathrm{e}}$ siècle $^{12}$, de véritables catalogues rédigés par les experts, marchands et restaurateurs. Si les considérations matérielles sont de plus en plus précisées, certains praticiens comme Jean-Louis Laneuville vont jusqu'à spécifier leurs restaurations, comme en 1851 à propos d'une grisaille de rentoiler ce tableau ${ }^{13}$. » Les mentions de « craquelures", « gerçures ", « repeints " et anciennes restaurations sur les supports sont plus récurrentes dans ces notices et soulignent l'intérêt grandissant des collectionneurs pour l'aspect matériel des ouvres.

Au même moment, les premiers catalogues avec notices détaillées des collections du musée du Louvre sont réalisés par Frédéric Villot, nommé conservateur des peintures à partir de 1849. Ils permettent au lecteur de connaître les différentes collections par lesquelles les œuvres sont passées avant d'entrer au musée, ainsi que d'appréhender l'état matériel du support et de la couche picturale des tableaux. Du domaine privé à l'espace public du musée, les données relatives aux mouvements des œuvres et à leur état de conservation représentent ainsi des informations qui intéressent de plus en plus les amateurs de peinture.

\section{Collectionneur et restaurateur : vers une reconnaissance des professions?}

Lorsque, en 1851, le restaurateur Simon Horsin-Déon commence par dédier son ouvrage De la conservation et de la restauration des tableaux «à Monsieur G. Doazan, Hommage d'estime et d'affection ", il célèbre d'une part la volonté du collectionneur de connaître et de comprendre les techniques employées par les peintres anciens, et salue d'autre part son désir d'être " initié aux mystères de la restauration ${ }^{15}$ ». On peut donc se demander dans quelle mesure la consultation des restaurateurs par les amateurs d'art a pu contribuer, parmi d'autres facteurs, à la reconnaissance de la profession de restaurateur au $\mathrm{XIX}^{\mathrm{e}}$ siècle.

Comme le rappelle Pascal Labreuche, c'est d'ailleurs la particularité de ce métier au XIX ${ }^{\mathrm{e}}$ siècle : la restauration ne se définit plus comme une "spécialisation ${ }^{16}$ " associée à une autre profession. Le restaurateur peut, toutefois, soit être qualifié de "réparateur » ou de «médecin ${ }^{17}$ ", soit au contraire être condamné pour avoir fait acte de «maladresse " et de «scélérate brutalité ". Jusqu'en 1801, les répertoires du commerce n'individualisent pas encore les professions et renvoient à «Tableaux. Marchands et

Restaurateurs de ". À partir de 1803, les restaurateurs de tableaux sont mentionnés indépendamment. Les mêmes almanachs citent, cette année-là, onze noms de restaurateurs $^{19}$, soulevant la question d'une division de la profession entre ceux rattachés ou non aux musées nationaux.

La frontière est d'ailleurs poreuse du côté des restaurations de collections privées réalisées par les ateliers du Louvre. En avril 1848, Philippe-Auguste Jeanron constate, à son arrivée au poste de directeur des musées nationaux, que « des tableaux étrangers au musée étaient restaurés officieusement dans les ateliers du Louvre. La nouvelle administration a trouvé, dans ce cas, 64 tableaux appartenant à plusieurs personnes $^{20} »$. Bien que les ateliers connaissent une réorganisation et la mise en place d'un concours des restaurateurs entre 1848 et $1850^{21}$, des particuliers continueront de faire appel aux restaurateurs du Louvre. C'est par exemple le cas d'Émile Mortemard qui, en 1858, restaure une toile appartenant à James de Rothschild ${ }^{22}$, ou encore en 1869, un tableau en possession d'un certain M. Martin²3.

\section{L'amateur : connaissance scientifique et rôle auprès des musées}

Parallèlement à la professionnalisation du métier de restaurateur, la diffusion d'écrits sur les techniques artistiques n'encourage-t-elle pas le collectionneur à intervenir directement sur la peinture, en ayant « la prétention d'améliorer une œuvre [...] privilège que se donne celui qui l'a acquise à prix d'argent ${ }^{24}$ »? La lecture de la littérature technologique de l'époque lui est d'ailleurs principalement destinée. Ainsi, dès 1808, François-Xavier Burtin dans son Traité théorique et pratique des connaissances qui sont nécessaires à tout amateur de tableaux consacre un chapitre aux « différentes Méthodes qu'on peut mettre en usage pour Nettoyer les Tableaux, et des précautions qu'on doit prendre en les faisant rentoiler ou restaurer » en soulignant l'utilité que présente le fait de nettoyer soi-même l'œuvre "en commençant par des essais sur quelques mauvais tableaux ${ }^{25} »$. Cette littérature, largement diffusée à Paris, favorise une meilleure connaissance des procédés techniques employés par les peintres et/ou restaurateurs.

Au milieu du siècle, les collectionneurs acquièrent d'ailleurs un rôle notable au sein de l'administration muséale au titre de leur expertise. Faisant suite au remaniement de l'administration et des ateliers du Louvre en 1848-1850, une commission de surveillance pour la restauration des tableaux des musées nationaux est mise en place. À côté des restaurateurs Simon Horsin-Déon et Étienne-François Haro, y sont nommés les amateurs messieurs La Caze, Collon et Tencé. En 1855, Louis La Caze, qui avait lui-même été peintre (fig. 2), est par ailleurs nommé au jury d'examen et d'admission des œuvres d'art de l'Exposition universelle et, encore en 1861, au sein de la Commission consultative des Musées impériaux. En 1869, il lègue sa collection au Louvre, dont cinq cent quatre-vingt-trois tableaux ${ }^{26}$. Le rôle progressif acquis par les 
collectionneurs au sein des commissions est ainsi lié à leur expertise en conservation, restauration et politique d'acquisition des musées.

$\mathrm{Si}$, au XIX $\mathrm{X}^{\mathrm{e}}$ siècle, le rapport entre l'amateur d'œuvres d'art et le restaurateur de tableaux permet de soulever différents questionnements quant au parcours entrepris par l'œuvre entre le(s) cabinet(s) privé(s) et le musée, cette thématique soulève des enjeux plus larges du point de vue social et culturel. En effet, l'amateur et le restaurateur de tableaux obtiennent progressivement des positions de conseillers au sein des commissions des Beaux-Arts en France, rôles enrichis par la complémentarité de leurs connaissances et de leurs échanges.

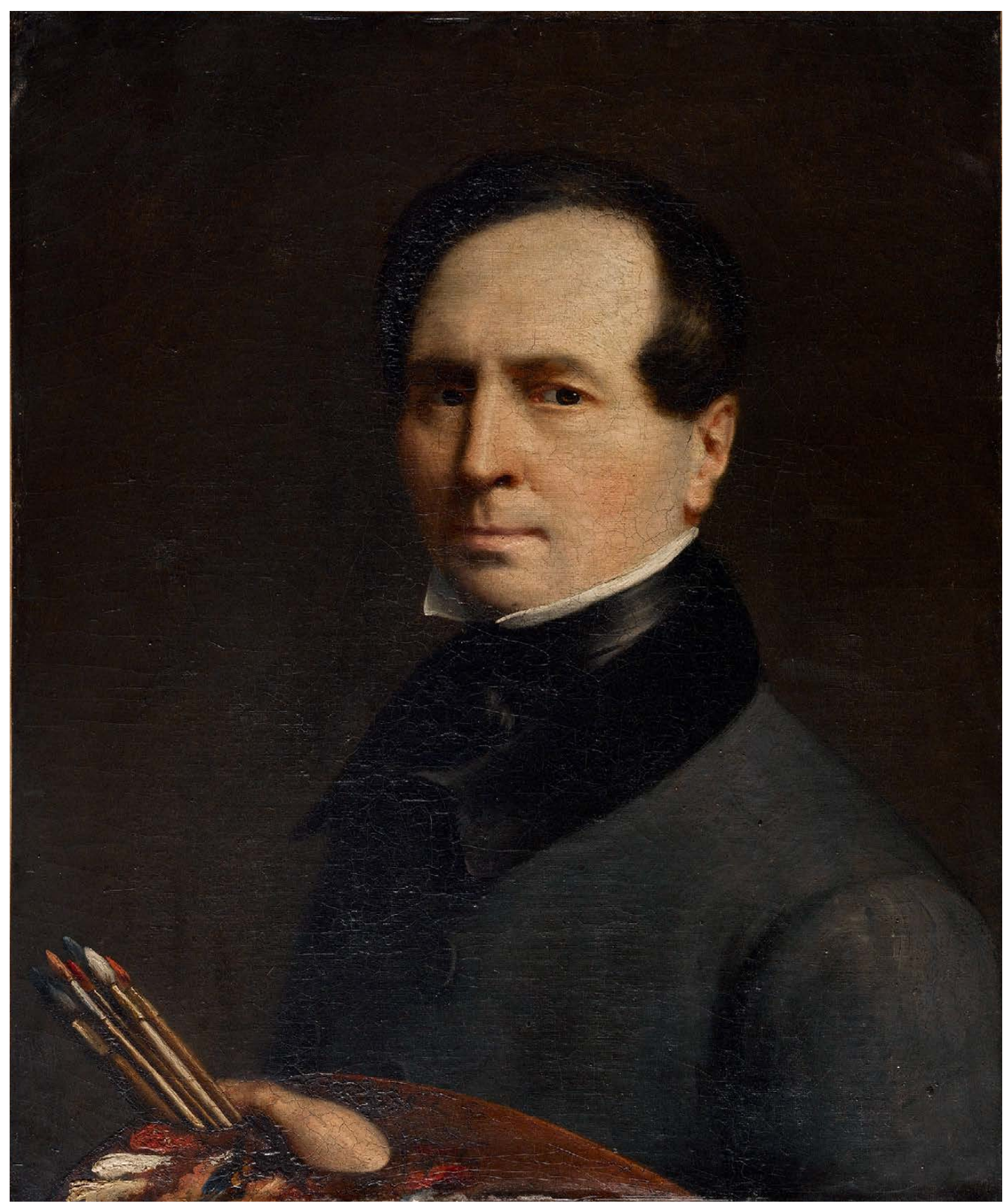

Fig. 2. Louis La Caze, Portrait de l'artiste, vers 1843, huile sur toile, Paris, musée du Louvre. (c) RMN-Grand Palais (musée du Louvre)/Franck Raux. 


\section{Notes}

1. Étienne, 2012 ; Massing, 2012 ; Émile-Mâle, 2008

2. Jouves, 2017

3. Thiéry, 1790.

4. Lacroix, 1861

5. Guichard, 2008 ; Michel, 2007.

6. Thèse en cours, depuis 2015 :

Barbara Jouves, Conservation et restauration des peintures des collections privées à Paris entre 1789 et 1870 [titre provisoire], sous la direction de Thierry Lalot, professeur des universités, université Paris 1 PanthéonSorbonne.

7. Assézat, 1876

8. Michel, 2010 ; Chatelus, 1991.

9. Poulot, 2001, p. 23 ; Pety, 2010.

10. Michel, 2002 ; Haskell, 1986.

11. L'ensemble des catalogues de ventes aux enchères de peintures pour la période 1789-1870 a été consulté à partir de : Lugt F., 1938 à 1954, t. I à III.

12. Pomian, 1987.

13. Vente des 19 et 20 mars 1851, p. 6 , $\mathrm{n}^{\circ} 5$ : la vente est réalisée par le ministère de $\mathrm{M}^{\mathrm{e}}$ Bonnefons de Lavialle, commissairepriseur, assisté de M. Ferdinand Laneuville, expert.

14. Villot, 1849.

15. Horsin-Déon, 1851

16. Labreuche, 2011

17. Golovine, 1862.

18. Tabarant, 1963 [1942].

19. Almanach du commerce de Paris, 1803 p. 204 : sont cités, par ordre alphabétique, les noms de Clisorius, Doussin, Fouque, Guillemard, Hacquin, Hooghstoels, Jeanteaux, Letourneur, Renard, Roeser et Sagedieu.

20. Jeanron, 1848.

21. Lauwick, Cabillic, Gerin-Pierre, 2008, p. 106-108.

22. Archives nationales, Archives des musées nationaux, 20144790/140 Logements et affectations de locaux (18241914). 1858/09/03 : M. Mortemard, restaurateur, est autorisé à effectuer dans une salle du Louvre la restauration d'une grande toile pour plafond appartenant à James de Rothschild. 23. Id., 1869/08/19 : Mortemard demande l'autorisation de restaurer dans son atelier du Louvre un tableau appartenant à M. Martin.

24. Guillerme, 1964

25. Burtin, 1808.

26. Faroult, 2007.

\section{Bibliographie}

Almanach du commerce de Paris, 1797-1837, Bottin Sébastien, Paris.

Annuaire général du commerce, de l'industrie, de la magistrature et de l'administration, 1838 1856, Firmin Didot Frères, Paris

Annuaire-almanach du commerce, de l'industrie, de la magistrature et de l'administration, 1857-1870, Commerce Didot-Bottin, Paris.

Assézat J. (éd.), 1876, «Salon de 1767 », Euvres complètes de Diderot, Garnier Frères, Paris, t. XI, p. 7.

Burtin F.-X., 1808, Traité théorique et pratique des connaissances qui sont nécessaires à tout amateur de tableaux, Bruxelles, vol. 1 , p. 382-383.

Catalogue d'une belle collection de tableaux anciens des Écoles Italienne, Espagnole, Flamande, Hollandaise et Française, faisant partie de la Galerie Étrangère de M. le Prince de ***, dont la vente aura lieu les mercredi 19 et jeudi 20 mars 1851, 1851, Imprimerie et lithographie Maulde et Renou, Paris.

Chatelus J., 1991, Peindre à Paris au XVIII siècle, Éditions Jacqueline Chambon, Nîmes.

Émile-Mâle G., 2008, Pour une histoire de la restauration des peintures en France, S. Bergeon-Langle (éd.), Éditions Somogy/Inp, Paris.

Étienne N., 2012, Les restaurations des peintures à Paris (1750-1815): Pratiques et discours sur la matérialité des ouvres d'art, Presses Universitaires de Rennes, Rennes.

Faroult G. (dir.), Eloy S., 2007, La collection La Caze. Chefs-d'œuvre des peintures des $X V I I^{e}$ et XVIII ${ }^{e}$ siècles, Éditions Hazan/ Éditions du musée du Louvre, Paris, p. 11.

Golovine I., 1862, Manuel du marchand de tableaux, E. Dentu, Paris, p. 27.

Guichard C., 2008, Les amateurs d'art à Paris au XVIII siècle, Champ Vallon, Seyssel.

Guillerme J., 1964, L'atelier du temps. Essai sur l'altération des peintures, Hermann, Paris, p. 47-48.

Haskell F., 1986, La Norme et le caprice, redécouvertes en art : aspects du goût de la mode et de la collection en France et en Angleterre (1789-1914), Flammarion, Paris.

Horsin-Déon S., 1851, De la conservation et de la restauration des tableaux. Éléments de l'art du restaurateur; historique de la partie mécanique de la peinture, depuis sa renaissance jusqu'à nos jours ; classification de toutes les écoles; recherches et notices sur quelques grands maîtres, Chez Hector Bossange, Paris, p. VI-VIII.
Jeanron P.-A., 28 avril 1848, « Rapport du 23 avril 1848 sur la situation des ateliers de restauration au musée du Louvre, et sur leur organisation ", Moniteur universel, p. 4

Jouves B. (dir.), 2017, Réception critique de la restauration $\mathrm{XVIII}^{e}-\mathrm{XIX} \mathrm{X}^{e}$ siècles. Actes de la journée d'étude (Paris, 27 octobre 2016), Éditions du GRHAM, Paris.

Labreuche P., 2011, Paris, capitale de la toile à peindre. XVIII ${ }^{e}-X X^{e}$ siècles, CTHS-INHA, Paris, p. 93.

Lacroix P., 1861, Annuaire des artistes et des amateurs, Jules Renouard, Paris, p. 107-116.

Lauwick B., Cabillic I., Gerin-Pierre C., 2008, «Les concours de restaurateurs de peinture au Louvre ", Technè, $n^{\circ}$ 27-28, p. 105-111.

Lugt F., 1938 à 1954, Répertoire des catalogues de ventes publiques intéressant l'art ou la curiosité, Martinus Nijhoff, La Haye.

Massing A., 2012, Painting restoration before $\mathrm{La}$ Restauration: the origins of the profession in France, H. Millet: the Hamilton Kerr Institute, London/Cambridge.

Michel P., 2007, Le Commerce du tableau à Paris dans la seconde moitié du XVIII siècle, Presses universitaires du Septentrion, Villeneuve-d'Ascq.

Michel P., 2010, Peinture et plaisir, les goûts picturaux des collectionneurs parisiens au XVIII $I^{e}$ siècle, Presses universitaires de Rennes, Rennes

Michel P. (éd.), 2002, Collections et marché de l'art en France au XVIII siècle. Actes de la $3^{\mathrm{e}}$ journée d'étude d'histoire de l'art moderne et contemporain de Gironde (Bordeaux, avril 2000), université Michel de Montaigne-Bordeaux 3 - Les cahiers du Centre François-Georges Pariset, Bordeaux, p. 153.

Pety D., 2010, Poétique de la collection au $X I X^{e}$ siècle : du document de l'historien au bibelot de l'esthète, Presses universitaires de Paris Ouest [Nanterre], p. 291.

Pomian K., 1987, Collectionneurs, amateurs et curieux. Paris-Venise : XVI $-X V I I^{e}$ siècles, Gallimard, Paris, p. 163-164.

Poulot Dominique, 2001, « La morale du musée : 1789-1830», Romantisme, n 112 consacré à la collection, p. 23-30.

Tabarant A., 1963 [1942], La vie artistique au temps de Baudelaire, [Mercure de France], Paris, p. 73.

Thiéry L.-V., 1790, Le voyageur à Paris. Extrait du Guide des Amateurs $\mathcal{E}^{\circ}$ des Étrangers Voyageurs à Paris, chez Gattey, Paris, p. $142-146$

Villot F., 1849, Notice des tableaux exposés dans les galeries du musée national du Louvre. $1^{\text {re }}$ partie, Écoles d'Italie, Vinchon, Paris, p. X-XI. 